\title{
A High-IIP3 Third-Order Elliptic Filter With Current-Efficient Feedforward-Compensated Opamps
}

\author{
Nagendra Krishnapura, Abhishek Agrawal, and Sameer Singh
}

\begin{abstract}
A low-distortion active filter is realized using currentefficient feedforward-compensated operational amplifiers in the integrators and feedforward current injection in the summing amplifier. A third-order elliptic low-pass filter with two possible bandwidth settings of 17 and $8.5 \mathrm{MHz}$ consumes $1.8 \mathrm{~mW}$ from a 1.8-V supply and occupies $0.17 \mathrm{~mm}^{2}$ in a $0.18-\mu \mathrm{m}$ CMOS process. The measured maximum signal-to-noise and distortion ratios at the two bandwidth settings are 50.5 and $52.5 \mathrm{~dB}$, respectively. The corresponding third-order intermodulation intercept points (IIP3) are +28.2 and $+30.8 \mathrm{dBm}$. Automatic tuning is used at the startup to counter process variations and set the bandwidth accurately.
\end{abstract}

Index Terms-Automatic tuning, continuous-time filters, elliptic filters, feedforward compensation.

\section{INTRODUCTION}

I $\mathrm{N}$ zero-intermediate-frequency integrated-circuit radio receivers, low-pass filters [1]-[3] are used before analog/ digital conversion. Their bandwidths range from a few megahertz to a few tens of megahertz. A switchable bandwidth is required for multistandard radios. For example, 802.11 [wireless local-area network (WLAN)] has twice the signal bandwidth in the " $\mathrm{n}$ " mode as in the "a, b, and g" modes [4]. Another important requirement for these filters is a low distortion performance, usually specified using the third-order intermodulation intercept point (IIP3). Among integrated continuous-time filters, active $R C$ filters provide the best distortion performance at medium frequencies because they consist of integrators and amplifiers using operational amplifiers (opamps) in negative feedback loops, which suppress the distortion arising from active elements. The distortion in these filters can be reduced by reducing the virtual-ground voltage swings, which are the "error" voltages of feedback loops around opamps. This can be achieved by increasing the gain of the opamp or reducing the output current driven by the opamp. For a given unity gain frequency and bias current, higher gain at low frequencies can be obtained using feedforward-compensated opamps [5] instead of their Miller-compensated counterparts. The output current driven from the opamp can be reduced by injecting a replica of the current to the output through a separate path [6]. Both these techniques lower the voltage variations at the virtual-ground nodes of opamps, making the operation of the feedback loop more ideal. This brief presents a low-distortion third-order elliptic filter that uses power-efficient feedforward-

Manuscript received July 8, 2010; revised October 19, 2010 and December 2, 2010; accepted February 11, 2011. Date of current version April 20, 2011. This paper was recommended by Associate Editor R. Martins.

The authors are with the Department of Electrical Engineering, Indian Institute of Technology, Madras, Chennai 600036, India (e-mail: nagendra@ iitm.ac.in).

Digital Object Identifier 10.1109/TCSII.2011.2124571

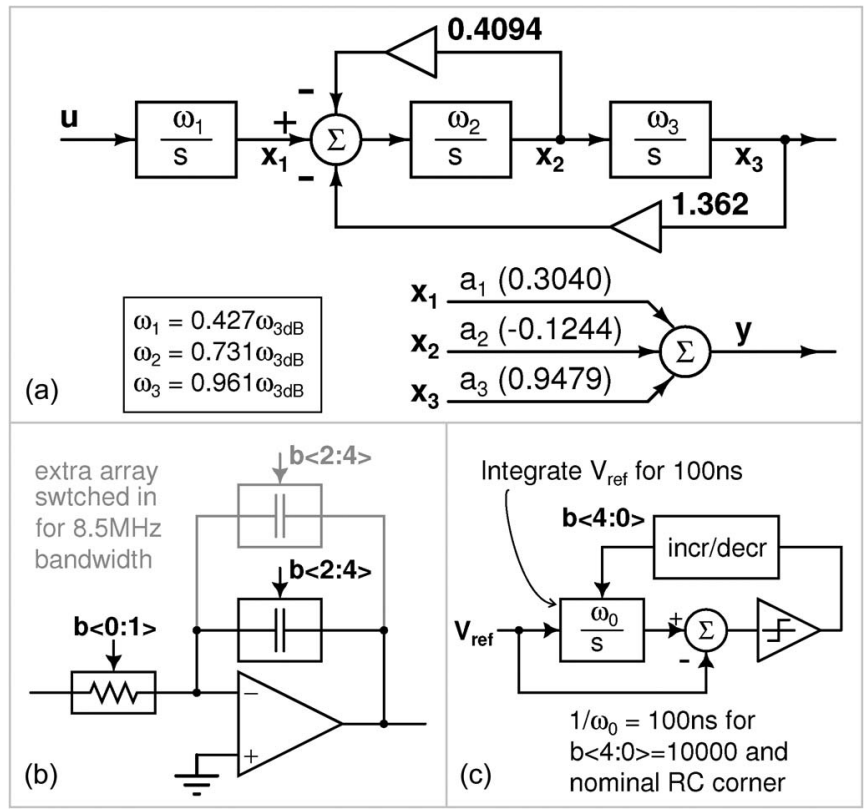

Fig. 1. (a) Filter block diagram. (b) Integrator realization (single-ended equivalent shown for clarity). (c) Automatic tuning scheme.

compensated opamps in the integrator and partial injection of the opamp output current in the summing amplifier. The filter incorporates automatic tuning to correct for process variations, and its bandwidth is programmable to 17 and $8.5 \mathrm{MHz}$ to cater to the two modes mentioned above.

This brief is organized as follows. Section II describes the filter architecture and automatic tuning and illustrates the advantages of using feedforward-compensated opamps in active- $R C$ filters. A current-efficient feedforward-compensated opamp is described in Section IV. The summing amplifier with current injection for the distortion reduction is described in Section V. The measured results are discussed in Section VI. Section VII concludes the brief.

\section{Third-Order ElLiptic FiLter}

Fig. 1(a) shows the block diagram of the third-order elliptic filter. The integrator outputs are scaled for equal maximum transfer function magnitude. Outputs of a cascade of first- and second-order sections are summed to realize the transmission zero. Fig. 1(b) shows the realization of each integrator. A 5-bit control word $b\langle 4: 0\rangle$ switches the resistors and the capacitors and varies the $R C$ product from $55 \%$ to $175 \%$ of the midcode value to compensate for process variations. Doubling the feedback capacitor array, as shown in Fig. 1(b), halves the bandwidth to $8.5 \mathrm{MHz}$ while maintaining the same passbandnoise spectral density as required in WLAN receivers. 
(a)



(b)

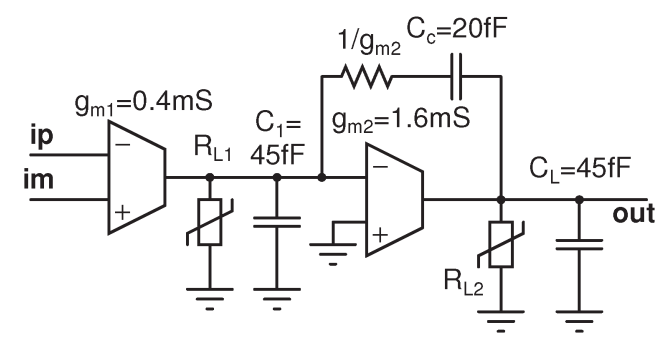

Fig. 2. Macromodel of (a) feedforward- and (b) Miller-compensated opamps (single-ended equivalents shown for clarity).

The automatic tuning scheme is shown in Fig. 1(c). It uses an additional integrator, which is a replica of Fig. 1(b). With nominal resistor and capacitor values, the replica integrator's time constant $1 / \omega_{0}$ is $100 \mathrm{~ns}$ when the digital bits are set to midcode. Across process variations, the time constant can be tuned to $100 \mathrm{~ns}$ by setting the five control bits. The appropriate control bits are generated as follows. The replica integrator is initially reset, and an internally generated reference voltage $V_{\text {ref }}$ (approximately $400 \mathrm{mV}$ ) is applied to it for a period of $100 \mathrm{~ns}$. The 100-ns interval is generated using an accurate external clock. At the end of the integration period, the output of the integrator is compared with $V_{\text {ref }}$. The tuning bits are updated based on the result of the comparison [7]. The integrator is reset, and the cycle repeats. After five cycles of successive approximation, the bits converge to a value that sets the time constant of the replica integrator to $100 \mathrm{~ns}$. The same bits are applied to all the integrators, and by the virtue of matching, their unity gain frequencies are set to their correct values. Due to the digital nature of tuning, there can be up to $5 \%$ error in the bandwidth. The tuning circuitry is disabled after the process is complete.

\section{AdVAnTAGES OF FEEDFORWARd OPAMPS}

Fig. 2 shows the macromodels of the feedforward- and Miller-compensated opamps. The first stage consists of $g_{m 1}$ loaded by $R_{L 1}$ and $C_{1}$, and the second stage consists of $g_{m 2}$ loaded by $R_{L 2}$ and $C_{L}$. In Fig. 2(a), the feedforward stage $g_{\mathrm{mf}}$ provides a high-speed path from the input to the output for frequency compensation [5]. In Fig. 2(b), $C_{c}$ (with zero canceling resistor $1 / g_{m 2}$ in series) is used across the second stage for frequency compensation [8]. The cascade of two stages using $g_{m 1}$ and $g_{m 2}$ provides a high gain in both cases. Table I compares the parameters of the two opamps. For stability, the nondominant pole $p_{2}$ of the Miller-compensated opamp has to be higher than its unity gain frequency $\omega_{u}$. Therefore, for a given unity gain frequency $\omega_{u}$ and a given load capacitance $C_{L}$, $g_{m 2}$ in the Miller-compensated opamp has to be significantly more than $g_{\mathrm{mf}}$ in the feedforward opamp. The input-referred noise voltage at low frequencies is determined only by the first stage $g_{m 1}$ in both cases.
TABLE I

COMPARISON BETWEEN FEEDFORWARD AND MILLER OPAMPS

\begin{tabular}{c|c|c}
\hline & Feedforward & Miller \\
\hline Unity gain frequency $\omega_{u}$ & $g_{m f} / C_{L}$ & $g_{m 1} / C_{c}$ \\
Non dominant pole $p_{2}$ & - & $p_{2}<g_{m 2} / C_{L}$ \\
Input ref. noise ( $\eta$ : a constant) & $\eta k T / g_{m 1}$ & $\eta k T / g_{m 1}$ \\
\hline
\end{tabular}
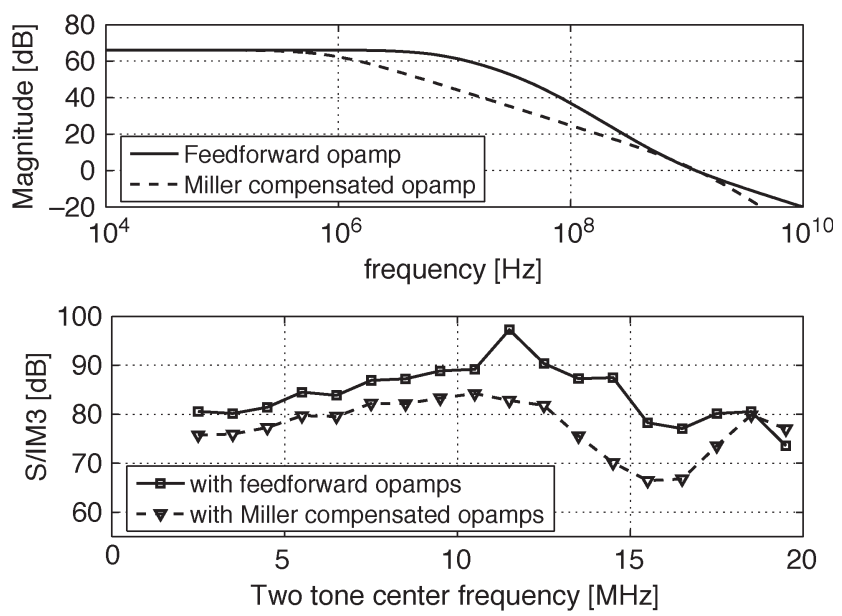

Fig. 3. (a) Magnitude response of the opamp models in Fig. 2. (b) S/IM3 of the elliptic filter using the opamp models in Fig. 2. The input consists of two tones $1 \mathrm{MHz}$ apart and with a $225-\mathrm{mV}$ peak each. Their center frequency is swept from 2.5 to $19.5 \mathrm{MHz}$

Fig. 3(a) shows the gain magnitude response of Miller- and feedforward-compensated opamps designed for a dc gain of $66 \mathrm{~dB}$ and a unity gain frequency of $1.8 \mathrm{GHz}$ while driving a load of $45 \mathrm{fF}$. The numerical values of all components are shown in Fig. 2. The first stage has a gain of about $40 \mathrm{~dB}$. The feedforward-compensated opamp has a significantly higher gain in a certain range of frequencies. This results in a lesser distortion when operated in a closed loop.

To verify this, the filter in Fig. 1(a) is realized using the opamps in Fig. 2 with weak nonlinearities added to transconductors and resistors. Transconductors are modeled as $i_{\text {out }, \mathrm{gm}}=I_{0} \tanh \left(v_{i, \mathrm{gm}} / V_{0}\right)$, and resistors are modeled as $i_{R}=I_{0} \tanh \left(v_{R} / V_{0}\right)$. The small signal (trans)conductance is given by $I_{0} / V_{0}$, and the value of $V_{0}$ determines the nonlinearity. In our models, $V_{0}$ is $100 \mathrm{mV}$ for $g_{m 1}, 225 \mathrm{mV}$ for $g_{m 2, f}$, and $600 \mathrm{mV}$ for $R_{L 1, L 2}$. The filter is simulated with the opamp macromodels in a circuit simulator, and the resulting ratio of the signal (S) to the third-order intermodulation distortion product (IM3) is shown in Fig. 3(b). The distortion of the filter with feedforward-compensated opamps is significantly lower than that with Miller-compensated opamps. The input-referred noise voltage of the two opamps is the same because the same value of $g_{m 1}$ is used for both. The total transconductance used in the Miller-compensated opamp is $2 \mathrm{mS}$, and that used in the feedforward-compensated opamp is $1.2 \mathrm{mS}$, implying proportionately smaller power consumption in the latter. We can therefore conclude that filters using feedforward-compensated opamps will have a better dynamic range for a given power dissipation.

\section{Current-EFFicient FEedforward OpAMP}

Fig. 4 shows the feedforward-compensated opamp proposed in [5]. A cascade of two n-channel MOS (nMOS) differential 


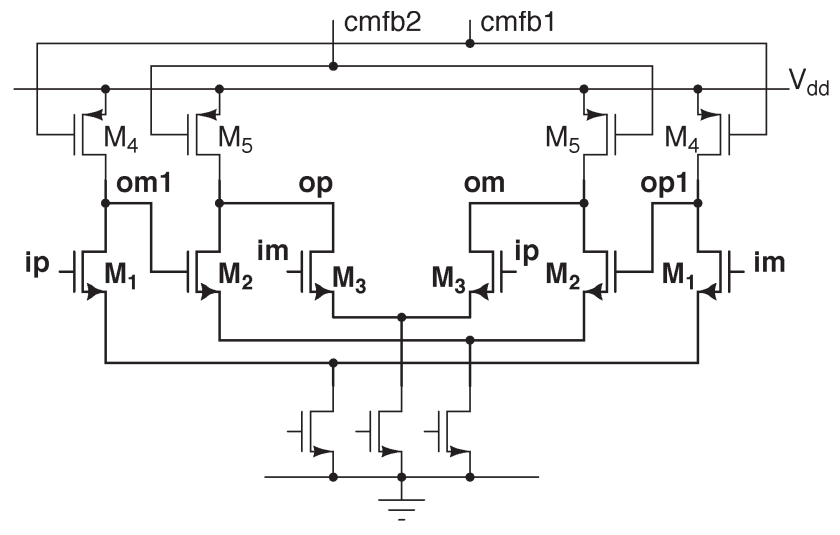

Fig. 4. Feedforward-compensated opamp in [5].

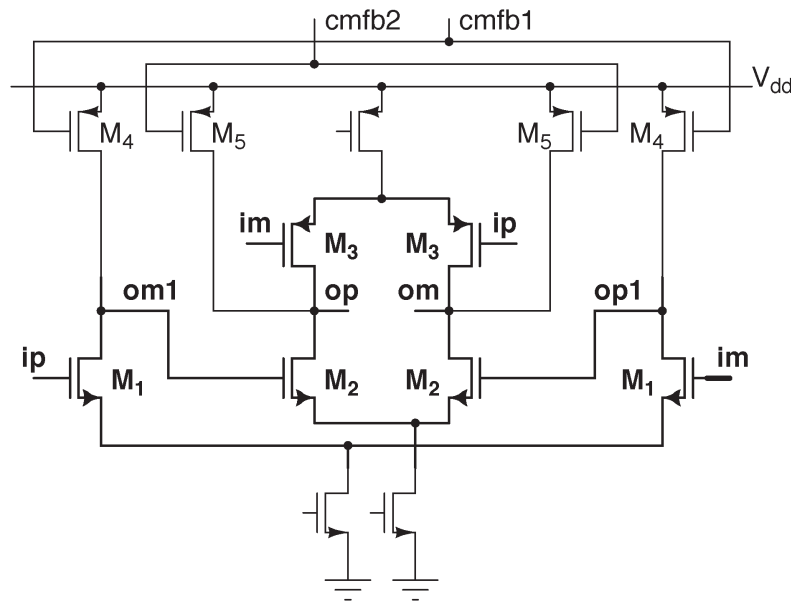

Fig. 5. Feedforward-compensated opamp with shared bias currents.

pairs $\left(M_{1}, M_{2}\right)$ provides a high gain. Feedforward compensation is provided by using another nMOS differential pair $M_{3}$.

A more current-efficient structure for feedforward compensation is to use a p-channel MOS differential pair $M_{3}$, which shares its bias current with the second-stage differential pair $M_{2}$, as shown in Fig. 5. Separate common-mode feedback circuit (CMFB) stages are used to drive current sources $M_{4}$ and $M_{5}$ and to stabilize the common-mode output of each stage. Fig. 6 shows the CMFB circuits used for the two stages. The common-mode voltage is fed back through $g_{m, \mathrm{~cm}}$ (a differential pair with a current mirror load). $C_{c}$ compensates the CMFB loop by providing a fast path to the gates of $M_{4}$ or $M_{5}$. For the first-stage CMFB loop [see Fig. 6(a)], source followers are used to drive the common-mode detector so that a high de gain is maintained. The opamp consumes about $0.2 \mathrm{~mA}$ and is used for all integrators and the summing amplifier in Fig. 1.

\section{SUMMing AMPLIFIER}

Fig. 7(a) shows the conventional summing amplifier. Because multiple inputs are summed, the loop gain tends to be lower, and the distortion tends to be higher than in an amplifier with a single input. With an ideal opamp, the virtual ground of the opamp is at zero. With a real opamp, the virtual ground experiences a signal swing proportional to $\left(a_{1} x_{1}+a_{2} x_{2}+a_{3} x_{3}\right) / R$, i.e., the current driven from the opamp. If the swing at the virtualground node is reduced, the weak nonlinearities of the opamp

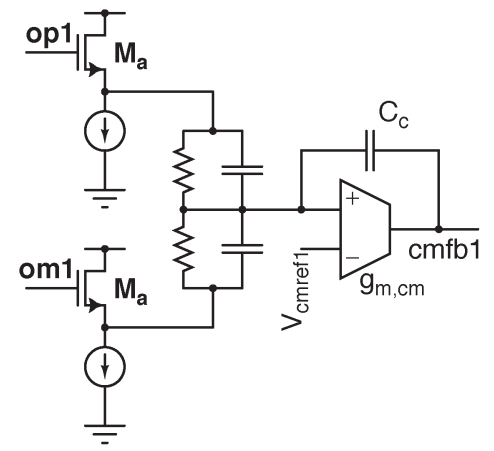

(a)

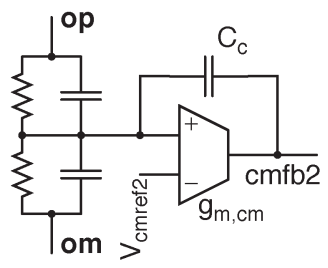

(b)
Fig. 6. CMFB for the (a) first and (b) second stages of the opamp in Fig. 5.

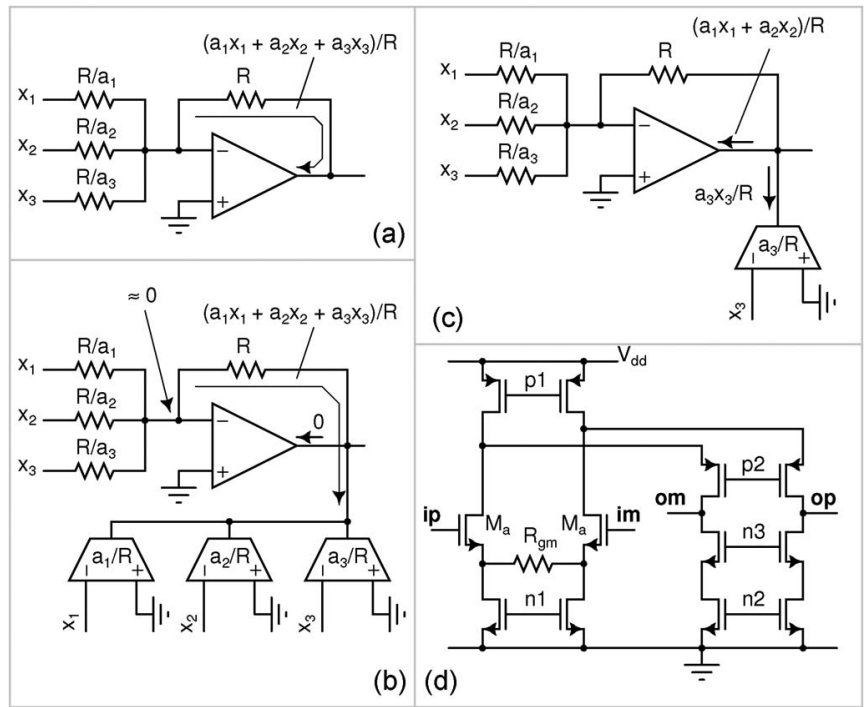

Fig. 7. (a) Conventional summing amplifier. (b) Summing amplifier with feedforward injection of the output current. (c) Our implementation with partial injection from only the largest contributor. (d) Transconductor implementation.

are exercised to a lesser extent, leading to lower distortion. Conventionally, this is done by increasing the gain of the opamp (at the relevant signal frequencies). Alternatively, the same can be accomplished by lowering the current driven from the opamp [6]. Fig. 7(b) shows the summing amplifier with a current $\left(a_{1} x_{1}+a_{2} x_{2}+a_{3} x_{3}\right) / R$ injected at the output. As the opamp has to deliver zero current, the virtual-ground voltage is zero, and the summing operation is ideal. In our implementation [see Fig. 7(c)], only $a_{3} x_{3} / R$, which is the largest component of the output current, is injected to the output using a transconductor. Fig. 7(d) shows the implementation of the transconductor used in Fig. 7(c). Its transconductance, which depends on $R_{\mathrm{gm}}$ and the transconductance of $M_{a}$, is adjusted to be equal to $a_{3} / R$ in the nominal process corner. For the same total bias current in Fig. 7(a) and (c), the latter has lesser distortion of 4 to $6 \mathrm{~dB}$ over process corners.

\section{Measured Results}

The third-order filter with automatic tuning is fabricated in a $0.18-\mu \mathrm{m}$ CMOS process. Fig. 8 shows the layout, the test schematic, and the test board. The buffers in Fig. 8(b) can be switched to have gains of $+A,-A$, or 0 (off). By determining the transfer function of the filter path and the buffer path 

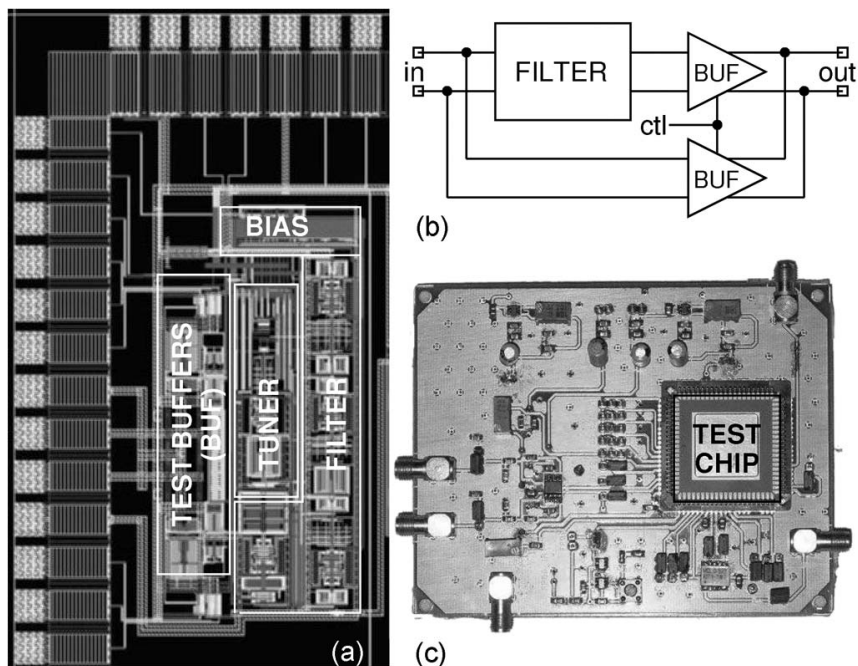

(b)

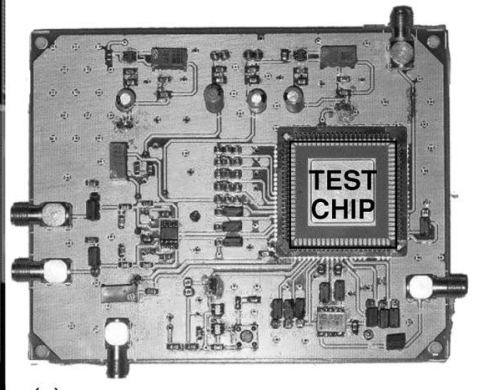

(c)

Fig. 8. (a) Chip layout. (b) Test schematic (the buffers are on chip). (c) Test board.

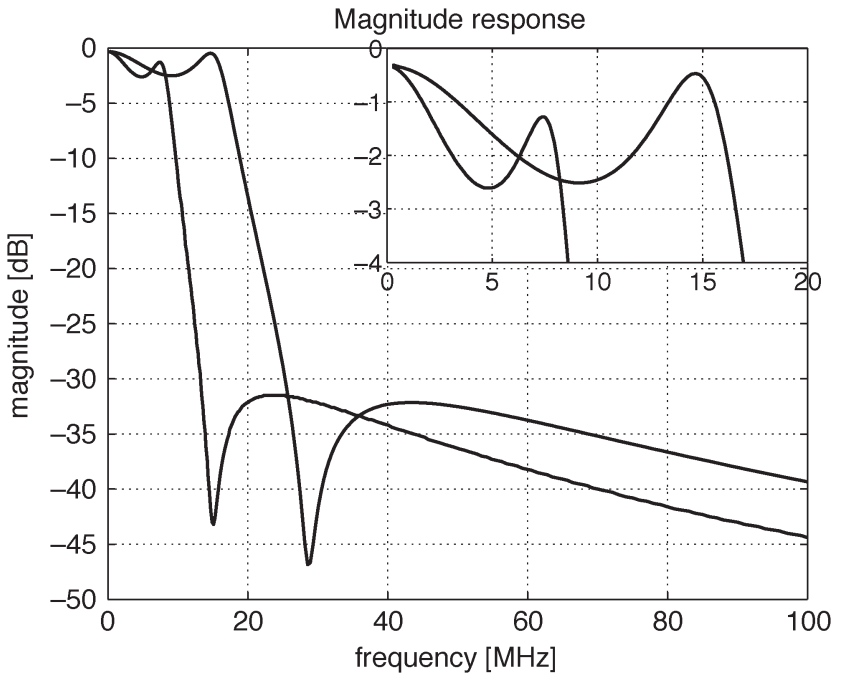

Fig. 9. Measured magnitude response for 17- and 8.5-MHz settings.

for both positive and negative settings of the buffer gain, the external feedthrough can be cancelled, and the transfer function of the filter can be accurately measured [9]. The measured magnitude response at $17-$ and $8.5-\mathrm{MHz}$ settings are shown in Fig. 9. The attenuation is more than $30 \mathrm{~dB}$ for frequencies more than $50 \%$ of the bandwidth. The maximum deviation of the inband group delay from the average value is $62 \mathrm{~ns}$ for the 8.5-MHz bandwidth and $45 \mathrm{~ns}$ for the 17-MHz bandwidth. In the small number of characterized samples, the 3 - $\mathrm{dB}$ bandwidth after automatic tuning is $16.3-16.6 \mathrm{MHz}$ in the high-bandwidth mode and 7.5-8.4 MHz in the low-bandwidth mode. The noise voltage integrated from 0.1 to $20 \mathrm{MHz}$ are $0.79 \mathrm{mV}$ for the $17-\mathrm{MHz}$ bandwidth and $0.68 \mathrm{mV}$ for the $8.5-\mathrm{MHz}$ bandwidth. The inband S/IM3 for two tones spaced $1 \mathrm{MHz}$ apart and with a combined strength of $+2 \mathrm{dBm}$ is shown in Fig. 10. As expected, the worst cases occur near the band edges due to peaking in the magnitude response at the internal nodes. The variation of S/IM3 with the amplitude is characterized by feeding two tones at the worst case frequencies and sweeping their amplitude. Fig. 11 shows the ratios of signal to noise, signal to IM3, and
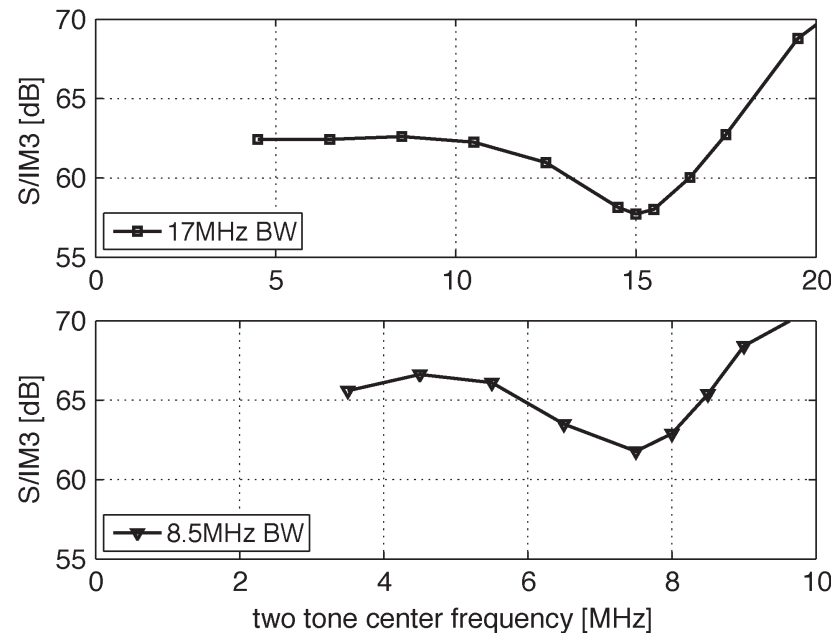

Fig. 10. S/IM3 versus frequency in the two bandwidth settings. The input consists of two sinusoids $1 \mathrm{MHz}$ apart and $-1 \mathrm{dBm}$ each.
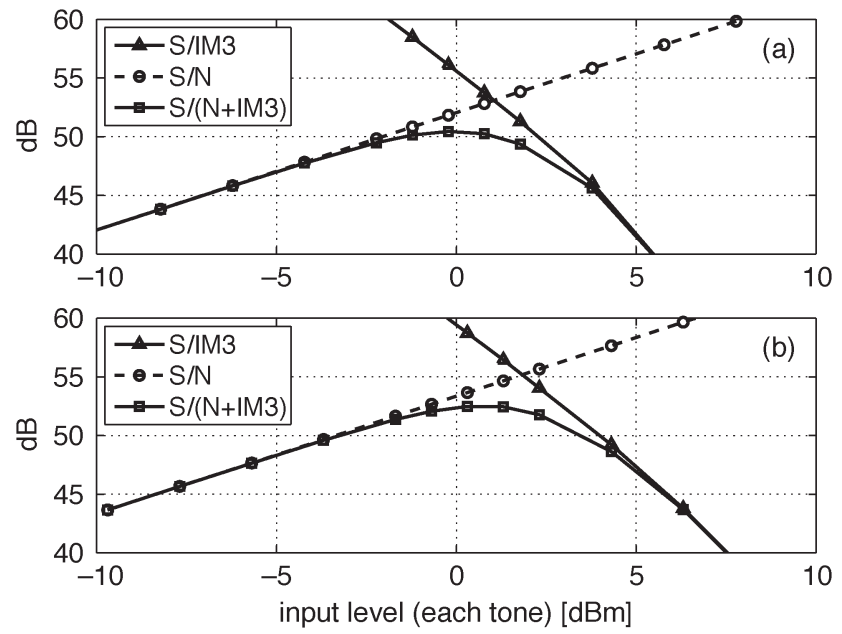

Fig. 11. Noise and distortion. (a) 17- and (b) 8.5-MHz bandwidth. The input consists of two tones at the worst case frequencies in Fig. 10.

TABLE II

PERFORMANCE SUMMARY

\begin{tabular}{c|c|c}
\hline Filter type & $2 \mathrm{~dB}$ passband ripple Elliptic \\
Order & \multicolumn{2}{|c}{3} \\
Technology/Supply voltage & \multicolumn{2}{|c}{$0.18 \mu \mathrm{m} \mathrm{CMOS} / 1.8 \mathrm{~V}$} \\
Area (filter/tuner) & $0.17 \mathrm{~mm}^{2} / 0.08 \mathrm{~mm}^{2}$ \\
Power (filter/tuner) & $1.8 \mathrm{~mW} / 0.49 \mathrm{~mW}$ \\
\hline$-3 \mathrm{~dB} \mathrm{BW}$ & $17 \mathrm{MHz}$ & $8.5 \mathrm{MHz}$ \\
DC gain & $-0.4 \mathrm{~dB}$ & $-0.4 \mathrm{~dB}$ \\
Passband ripple & $2.2 \mathrm{~dB}$ & $2.2 \mathrm{~dB}$ \\
Noise (0.1 MHz to 20 MHz) & $790 \mu \mathrm{V}$ & $680 \mu \mathrm{V}$ \\
Noise PSD (in band) & $170 \mathrm{nV} / \sqrt{\mathrm{Hz}}$ & $170 \mathrm{nV} / \sqrt{\mathrm{Hz}}$ \\
SNDR $\max$ & $50.5 \mathrm{~dB}$ & $52.5 \mathrm{~dB}$ \\
FOM & $0.25 \mathrm{fJ}$ & $0.3 \mathrm{fJ}$ \\
IIP3 (worst case in band) & $28.2 \mathrm{dBm}$ & $30.8 \mathrm{dBm}$ \\
\hline \multicolumn{2}{|c}{}
\end{tabular}

signal to the sum of noise and IM3 [signal-to-noise and distortion ratios (SNDRs)] versus the input level. The worst case inband IIP3 values at the high- and low-bandwidth settings are +28.2 and $+30.8 \mathrm{dBm}$, respectively. The maximum SNDRs are $50.5 \mathrm{~dB}$ for the $17-\mathrm{MHz}$ bandwidth and $52.5 \mathrm{~dB}$ for the 8.5-MHz bandwidth. The filter consumes $1 \mathrm{~mA}$, and the automatic tuning circuit consumes $270 \mu \mathrm{A}$ from a 1.8 -V supply. Table II summarizes the performance of the chip. 
TABLE III

COMPARISON With PUBLISHED FILTERS

\begin{tabular}{c|c|c|c|c|c|c|c|c}
\hline & This Work & This Work & {$[1]$} & {$[2]$} & {$[3]$} & {$[10]$} & {$[11]$} & {$[12]$} \\
\hline Type & Elliptic & Elliptic & Chebyshev & Bessel & Chebyshev & Butterworth & Bessel & Elliptic \\
Circuit & Active-RC & Active-RC & Active-RC & Gm-C & Gm-C & Gm-C & Source follower & Active-RC \\
\hline Order & 3 & 3 & 5 & 4 & 5 & 3 & 4 & 3 \\
Bandwidth $f_{B}$ & $17 \mathrm{MHz}$ & $8.5 \mathrm{MHz}$ & $19.7 \mathrm{MHz}$ & $11 \mathrm{MHz}$ & $9.2 \mathrm{MHz}$ & $2.2 \mathrm{MHz}$ & $10 \mathrm{MHz}$ & $10 \mathrm{MHz}$ \\
Attenuation at $2 f_{B}$ & $32 \mathrm{~dB}$ & $32 \mathrm{~dB}$ & $40 \mathrm{~dB}$ & $11 \mathrm{~dB}$ & $37 \mathrm{~dB}$ & $8 \mathrm{~dB}$ & $10 \mathrm{~dB}$ & $25 \mathrm{~dB}$ \\
Power $P_{d}$ & $1.8 \mathrm{~mW}$ & $1.8 \mathrm{~mW}$ & $11.25 \mathrm{~mW}$ & $14.2 \mathrm{~mW}$ & $15 \mathrm{~mW}$ & $1.92 \mathrm{~mW}$ & $4.1 \mathrm{~mW}$ & $4.6 \mathrm{~mW}$ \\
Integrated noise & $790 \mu \mathrm{V}$ & $680 \mu \mathrm{V}$ & $133 \mu \mathrm{V}$ & $36 \mu \mathrm{V}$ & $79 \mu \mathrm{V}$ & $96 \mu \mathrm{V}$ & $24 \mu \mathrm{V}$ & $267 \mu \mathrm{V}$ \\
IIP3 & $28.2 \mathrm{dBm}$ & $30.8 \mathrm{dBm}$ & $18.3 \mathrm{dBm}$ & $18 \mathrm{dBm}$ & $18.6 \mathrm{dBm}$ & $16.3 \mathrm{dBm}$ & $12 \mathrm{dBm}$ & $20.1 \mathrm{dBm}$ \\
\hline $2 / 3$ (IIP3 -N) & $51.5 \mathrm{~dB}$ & $53.7 \mathrm{~dB}$ & $55.2 \mathrm{~dB}$ & $62.6 \mathrm{~dB}$ & $58.4 \mathrm{~dB}$ & $55.8 \mathrm{~dB}$ & $60.9 \mathrm{~dB}$ & $52.4 \mathrm{~dB}$ \\
\hline FOM & $0.25 \mathrm{fJ}$ & $0.3 \mathrm{fJ}$ & $0.34 \mathrm{fJ}$ & $0.18 \mathrm{fJ}$ & $0.47 \mathrm{fJ}$ & $0.77 \mathrm{fJ}$ & $0.083 \mathrm{fJ}$ & $0.89 \mathrm{fJ}$ \\
\hline Area & $0.17 \mathrm{~mm}^{2}$ & $0.17 \mathrm{~mm}^{2}$ & $0.2 \mathrm{~mm}^{2}$ & $0.9 \mathrm{~mm}^{2}$ & $0.38 \mathrm{~mm}^{2}$ & $0.5 \mathrm{~mm}^{2}$ & $0.26 \mathrm{~mm}^{2}$ & $0.25 \mathrm{~mm}^{2}$ \\
Vdd & $1.8 \mathrm{~V}$ & $1.8 \mathrm{~V}$ & $1.5 \mathrm{~V}$ & $1.8 \mathrm{~V}$ & $3 \mathrm{~V}$ & $1 \mathrm{~V}$ & $1.2 \mathrm{~V}$ & $1 \mathrm{~V}$ \\
Process & $0.18 \mu \mathrm{m}$ & $0.18 \mu \mathrm{m}$ & $0.13 \mu \mathrm{m}$ & $0.18 \mu \mathrm{m}$ & $0.25 \mu \mathrm{m}$ & $0.18 \mu \mathrm{m}$ & $0.13 \mu \mathrm{m}$ & $0.12 \mu \mathrm{m}$ \\
& & & & & & & & \\
\hline
\end{tabular}

\section{CONCLUSION}

Power-efficient feedforward-compensated opamps in the integrators and feedforward current injection in the summing amplifier enable filters with an inherently high IIP3. To compare this filter to other continuous-time low-pass filters in the literature, we have used the fundamental relationship between the power and the bandwidth and the signal-to-noise ratio (SNR). In a first-order passive $R C$ filter, the ratio of the power dissipated $P_{d}$ to the product of the bandwidth $f_{B}$ and the SNR is a fundamental constant [13], i.e., $P_{d} /\left(f_{B} \times 10^{\mathrm{SNR} / 10}\right)=$ $2 \pi k T$, where the SNR is in decibels. There is no distortion in a passive filter and, hence, no upper limit to the signal amplitude. The power dissipation depends on the signal level. In an active filter, distortion places an upper limit on the input amplitude. The power dissipation is usually independent of the signal level. A figure of merit (FOM) inspired by the above relationship is the ratio of the power dissipated to the product of order $n$, bandwidth $f_{B}$, and the SNR at an input amplitude for which IM3 is equal to the noise level, ${ }^{1}$ and it is given by

$$
\mathrm{FOM}=P_{d} /\left(n \times f_{B} \times 10^{[2 / 3(\mathrm{IIP} 3-N)] / 10}\right) .
$$

Table III compares ${ }^{2}$ the filter described here to other published filters designed for similar bandwidths. The FOM and $2 / 3($ IIP3 $-\mathrm{N})$ are shown for each filter. The table also lists the attenuation at twice the filter's bandwidth as a measure of the width of the transition band. The IIP3 of the filter described here is at least $8 \mathrm{~dB}$ more than that of the others. For 17- and 8.5-MHz bandwidth settings, the FOM of the filters described here are 0.2 and $0.3 \mathrm{fJ}$. The FOM of the filters in [2] and [11] are lower, but these filters have a much broader transition band. The FOM of the filters presented here is better than those that have a comparably narrow transition band.

\section{REFERENCES}

[1] S. Kousai, M. Hamada, R. Ito, and T. Itakura, "A $19.7 \mathrm{MHz}$, fifth-order active- $R C$ Chebyshev LPF for draft IEEE802.11n with automatic quality-factor tuning scheme," IEEE J. Solid-State Circuits, vol. 42 , no. 11, pp. 2326-2337, Nov. 2007.

[2] S. D'Amico, V. Giannini, and A. Baschirotto, "A 4th-order active-Gm$R C$ reconfigurable (UMTS/WLAN) filter," IEEE J. Solid-State Circuits, vol. 41, no. 7, pp. 1630-1637, Jul. 2006.

[3] B. Shi and W. Shan, "A Gm-C baseband filter with automatic frequency tuning for a direct conversion IEEE802.11a wireless LAN receiver," in Proc. 30th Eur. Solid-State Circuits Conf., Sep. 2004, pp. 103-106.

[4] F. Horlin and A. Bourdoux, Digital Compensation for Analog Front Ends. New York: Wiley, 2008, p. 186.

[5] J. Harrison and N. Weste, "A $500 \mathrm{MHz}$ CMOS anti-alias filter using feedforward op-amps with local common-mode feedback," in Proc. IEEE Int. Solid-State Circuits Conf., Feb. 2003, vol. 1, pp. 132-483.

[6] S. Pavan and P. Sankar, "A $110 \mu \mathrm{W}$ single bit audio continuous-time oversampled converter with $92.5 \mathrm{~dB}$ dynamic range," in Proc. Eur. SolidState Circuits Conf., Sep. 2009, pp. 320-323.

[7] J. Lim, Y. Cho, K. Jung, J. Park, J. Choi, and J. Kim, "A wide-band active- $R C$ filter with a fast tuning scheme for wireless communication receivers," in Proc. IEEE Custom Integr. Circuits Conf., Sep. 2005, pp. 637-640.

[8] B. Razavi, Design of Analog CMOS Integrated Circuits. New York: McGraw-Hill, 2000.

[9] S. Pavan and T. Laxminidhi, "Accurate characterization of integrated continuous-time filters," IEEE J. Solid-State Circuits, vol. 42, no. 8 , pp. 1758-1766, Aug. 2007.

[10] T. Lo and C. Hung, "Low-voltage multi-mode Gm-C channel selection filter for mobile applications," in Proc. IEEE Custom Integr. Circuits Conf., Sep. 2007, pp. 635-638.

[11] S. D'Amico, M. Conta, and A. Baschirotto, "A 4.1-mW 10-MHz fourth-order source-follower-based continuous-time filter with $79-\mathrm{dB}$ DR,” IEEE J. Solid-State Circuits, vol. 41, no. 12, pp. 2713-2719, Dec. 2006.

[12] A. Vasilopoulos, G. Vitzilaios, G. Theodoratos, and Y. Papananos, "A lowpower wideband reconfigurable integrated active- $R C$ filter with $73 \mathrm{~dB}$ SFDR," IEEE J. Solid-State Circuits, vol. 41, no. 9, pp. 1997-2008, Sep. 2006.

[13] E. Vittoz, "Low power low-voltage limitations and prospects in analog design," in Analog Circuit Design, Low-Power, Low-Voltage, Integrated Filters and Smart-Power, R. J. V. D. Plassche, W. Sansen, and J. Huijsing, Eds. Boston, MA: Kluwer, 1995.

\footnotetext{
${ }^{1}$ At an input signal level equal to the IIP3, the IM3 (extrapolated from smallsignal levels) is also equal to the IIP3. Since the third-order distortion increases at $3 \mathrm{~dB}$ for a $1-\mathrm{dB}$ increase in the input signal, it can be worked out that the SNR is equal to $2 / 3($ IIP3 $-N$ ) when the distortion is equal to noise (both the IIP3 and $N$ are in decibels).

${ }^{2}$ Where the integrated noise is not reported, it is calculated by multiplying the inband spectral density by the square root of the bandwidth. Because of the noise peaking near the band edge, this underestimates the noise to some extent. The IIP3 shown is the worst case inband.
} 